\title{
Investigação das propriedades psicométricas de uma escala de metas de realização
}

\author{
Investigation of the achievement goals \\ scale psychometrics properties
}

\author{
José Maurício Haas BUENO ${ }^{1,2}$ \\ Rita da Penha Campos ZENORINI ${ }^{2}$ \\ Acácia Aparecida Angeli dos SANTOS ${ }^{2}$ \\ Alice Yuriko MATUMOTO \\ Juliana BUCHATSKY ${ }^{3}$
}

\section{Resumo}

A motivação para aprender é um fenômeno complexo e relevante, muito discutido na literatura estrangeira, mas pouco explorado no Brasil, principalmente em relação à sua mensuração. O objetivo deste trabalho foi investigar a estrutura fatorial, a precisão e as correlações entre os fatores de uma escala de metas de realização. Os participantes foram 156 estudantes de Psicologia, de ambos os sexos, com 22,3 anos em média, de duas universidades paulistas. Por meio de análise fatorial extraíram-se quatro fatores com precisões de 0,57 a 0,86. O primeiro fator foi relacionado à meta performance-aproximação, o segundo à meta aprender e o terceiro e quarto fatores a aspectos distintos da meta performance-evitação. Com base na análise das cargas fatoriais, correlações entre os fatores e estruturas lingüísticas das frases, foram feitas sugestões que devem auxiliar na continuidade do trabalho para obtenção de uma escala validada e fidedigna para avaliação das metas de realização.

Unitermos: análise fatorial; avaliação psicológica; propriedades psicométricas; teste de validade.

\begin{abstract}
The motivation to learn is a complex and relevant phenomenalargely discussed by the foreign literature, but not very much explored in Brazil. Thus, the objective of this study was to investigate the factorial structure, the reliability and the correlations among the factors of a goals achievement scale. Subjects were 156 undergraduate students of Psychology from two universities of São Paulo State, of both genders, whose mean age was 22.3 years. A factorial analysis extracted four factors with reliabilities varying from 0.573 to 0.860 . First factor was related to performance approach goal, the second to learning goal, and the third and fourth factors were related to distinct aspects of performance avoidance goal. Based on the factorial loadings, correlations between factors and linguistic structure phrases, suggestions were made to help other studies to obtain a valid and reliable scale to assess the achievement goals.
\end{abstract}

Uniterms: factor analysis; psychological assessment; psychometric properties; test validity.

४VVV

1 Universidade Presbiteriana Mackenzie. São Paulo, SP, Brasil.

2 Universidade São Francisco, Programa de Pós-Graduação Stricto Sensu em Psicologia. R. Alexandre Rodrigues Barbosa, 45, Centro, 13251-900, Itatiba, SP, Brasil. Correspondência para/Correspondence to: J.M.H. BUENO.E-mail: <jmhbueno@uol.com.br>.

3 Psicólogas, graduadas pela Universidade Presbiteriana Mackenzie. São Paulo, SP, Brasil. 
A motivação para aprender é um fenômeno bastante complexo e relevante, tornando-se um assunto bastante discutido na literatura estrangeira, mas que no Brasil necessita ser mais explorado. Como bem aponta Ruiz (2005), a sua relevância pode ser apreciada por diferentes ângulos, tendo em vista que esse é um tema incluído entre as principais preocupações de estudiosos direta ou indiretamente ligados à Psicologia e à Educação.

Pintrich e Schunk (2002) enfatizam que a motivação deve ser encarada como um processo e não um produto, embora só seja possível estudá-la pelos comportamentos que ela produz. Eles ressaltam que a motivação envolve objetivos ou metas que requerem esforço, persistência, planejamento, tomada de decisões, solução de problemas, entre outros, além de exigir o conhecimento de como cada um responde às dificuldades, problemas e fracassos encontrados. Os autores chamam a atenção para o fato de que são as expectativas, atribuições e sentimentos que irão ajudar o sujeito a superar as dificuldades e sustentar a motivação.

A teoria de metas de realização surgiu no final da década de 1970 e tem contribuído para o entendimento dos fatores motivacionais que influenciam o comportamento do aluno. $O$ conceito de meta refere-se ao aspecto qualitativo do envolvimento do aluno com situações de aprendizagem, expressando em nível hierárquico o propósito ou o porquê de uma pessoa envolver-se em uma tarefa. Assim, a meta de realização que a pessoa adota representa o motivo ou a razão pela qual ela irá realizar determinada tarefa. Essa teoria vem sendo utilizada amplamente em pesquisas estrangeiras que buscam compreender como os estudantes pensam em si próprios, nas suas tarefas e no seu desempenho (Ames, 1992; Anderman \& Maehr, 1994; Dweck\& Leggett, 1988; Elliott\&Dweck, 1988; Maehr \& Midgley, 1991; Meece, Blumenfeld \& Hoyle, 1988; Weiner, 1990).

O termo "metas de realização" é definido por Ames (1992) como um conjunto de pensamentos, crenças, propósitos e emoções que traduzem as expectativas dos alunos ao realizarem determinadas tarefas, o que significa que as metas são representadas por modos diferentes de enfrentar as atividades acadêmicas. A autora ressalta que quando o aluno desenvolve a crença de que essas metas têm valor e são significativas, bem como quando percebe que suas ações contribuem 80 para alcançar os objetivos pretendidos, ele passa a direcionar seu comportamento cognitivo e emotivo à realização.

Dois tipos básicos de metas são focalizados nas pesquisas com base na teoria de metas de realização, a saber: a meta aprender e a meta performance (Dweck \& Elliott, 1983; Dweck \& Leggett, 1988). Na literatura da área também são encontradas outras denominações, dependendo do autor que as refere, como envolvimento na tarefa e envolvimento do ego (Duda \& Nicholls, 1992; Nicholls, 1984) e meta domínio e meta performance (Ames, 1992, Ames \& Archer, 1988).

O aluno caracterizado pela meta aprender é aquele que acredita que os resultados positivos das atividades acadêmicas são decorrentes de seu esforço, que é um fator interno e sob seu controle. Em razão disso, direciona mais energia às suas atividades, enfrenta os desafios acadêmicos, interpreta os erros como um alerta para adotar novas estratégias e como uma oportunidade de crescimento, utiliza estratégias cognitivas e metacognitivas, o que se aproxima muito do conceito de motivação intrínseca (Ames, 1992).

Já o aluno caracterizado pela meta performance necessita comprovar a sua capacidade, sente necessidade de se mostrar inteligente, de se destacar entre os colegas ou de não parecer incapaz, crê que sua capacidade é evidenciada pelo fato de fazer melhor que os outros. Dessa forma, valoriza o reconhecimento público de que foi mais capaz que os demais. Assim, prefere tarefas nas quais possa se sobressair; encara os erros como falta de capacidade, apresentando sentimentos negativos diante do fracasso (Ames, 1992; Anderman \& Maehr, 1994; Dweck\&Elliot, 1983; Maehr\&Midgley, 1991).

Bzuneck (1999) observa que os dados referentes à meta aprender têm recebido comprovação em pesquisas mais recentes, proporcionando maior consistência histórica aos resultados comprovados dessa meta. No entanto isso não acontece com a meta performance. Muitas pesquisas apontam para seus efeitos prejudiciais enquanto outras descobriram nela aspectos positivos, principalmente quando acompanhada da orientação à metaaprender.

Nesse sentido, Elliot e Harackiewicz (1996); Skaalvik (1997) e Urdam (1997) identificaram dois componentes independentes na meta performance, quais sejam: o de aproximação (buscar parecer inteligente ou de ser o primeiro) e o de evitação (não querer parecer incapaz). Tais resultados demonstraram 
que o aspecto negativo da meta performance pode estar restrito ao componente de evitação. Autores como Bouffard, Boisvert, Vezeau e Larouche (1995) acreditam que a orientação adequada é aquela na qual o estudante não está preocupado somente com o conhecimento, mas também em alcançar e demonstrar um desempenho melhor.

Estudos que questionam os aspectos positivos da meta performance aproximação, como o de Midgley, Kaplan e Middleton (2001), referem-se a evidências de que essa meta é encontrada mais freqüentemente em meninos do que em meninas, em estudantes mais velhos do que nos mais jovens, e em ambientes de aprendizagem competitivos. Os autores questionam se, diante do fracasso, o aluno orientado à meta performance aproximação pode tender à meta performance evitação.

Em um trabalho de revisão sobre essa teoria, Harackiewicz, Barron, Pintrich, Elliot eThrash (2002) destacam os benefícios da meta performance aproximação associada à meta aprender. Os autores apontam três razões importantes para essa revisão: (a) a importância de separar os componentes aproximação e evitação da meta performance (Elliot, 1999; Elliot \& Harackiewicz, 1996; Pintrich, 2000); (b) o potencial positivo da meta performance aproximação em relação à meta aprender (Harackiewicz, Baron \& Elliot, 1998) e (c) a identificação de modelos nos quais a meta performance aproximação se combina com a meta aprender para promover uma ótima motivação (Barron \& Harackiewicz, 2000, 2001; Pintrich, 2000). Essas pesquisas endossam uma perspectiva de que é importante a aceitação da existência de metas múltiplas para a compreensão da motivação para a realização.

Grante Dweck (2003) consideram que os estudos que abordam as metas de realização têm esclarecido os processos básicos da motivação, ressaltando que há controvérsias com relação aos impactos dessas metas. Relatam um estudo longitudinal realizado em um curso de preparação de alunos para o curso de medicina, cujo resultado demonstrou que o impacto das metas aprender e performance depende de como elas são operacionalizadas. Os resultados obtidos indicaram a meta aprender como uma forma ativa de lidar com situações desafiadoras e a meta performance associada à desistência diante de dificuldades e a um desempenho mais baixo.

Com o objetivo de investigar os três tipos de metas - aprender, performance aproximação e perfor- mance evitação - e suas relações com exames de alunos não graduados, McGregor e Elliot (2002) encontraram um modelo indicador diferencial para cada uma das metas. A meta aprender relacionou-se com processos positivos (enfrentamento de desafios, concentração na preparação para uma atividade, por ex.), a meta performance aproximação apresentou-se relacionada a um conjunto mais limitado de processos positivos (avaliações dos desafios, aspiração às notas), e a meta performance-evitação relacionou-se a processos negativos (análise de ameaças, ansiedade antecipada a exames).

A motivação do estudante do ensino superior é um tema que vem sendo estudado por alguns pesquisadores brasileiros, tais como: Boruchovitch e Costa, (2001), Boruchovitch e Martini (1997), Bzuneck (1999, 2001), Cardoso (2002), Gombi (1999), Guimarães (2001, 2003), Guimarães, Bzuneck e Sanches (2002), Zenorini e Santos (2003), Ruiz (2005). Essas pesquisas utilizam diferentes enfoques, entre os quais a teoria de metas de realização.

O estudo realizado por Zenorini, Santos e Bueno (2003) buscou estabelecer evidências de validade da Escala de Sensibilidade às Diferentes Metas de Realização, desenvolvida por Midgley et al. (1998), que inclui os três tipos de metas referidas. Os autores identificaram a presença de um índice de precisão satisfatório para a meta aprender $(\alpha=0,72)$ e para a meta performance $(\alpha=0,84)$. No entanto, quando submetidas à análise fatorial, as metas se agruparam diferentemente do previsto, separando os itens em apenas dois fatores (meta aprender e meta performance). Os autores ressaltam que uma possível interpretação pode estar no fato de os sujeitos não terem percebido diferenças nos componentes aproximação e evitação da meta performance, impossibilitando a análise do aspecto positivo desse tipo de meta.

Os resultados encontrados por Zenorini et al. (2003) foram consistentes com os encontrados em outras pesquisas e revelaram uma alta correlação entre a meta aprendere o uso de estratégias de aprendizagem mais complexas. No entanto a meta aprender não apresentou correlação significativa com o desempenho acadêmico dos alunos. Um outro dado importante encontrado no estudo foi a diferença significativa na variável gênero. As participantes do sexo feminino mostraram-se mais orientadas à meta aprender, utili- 
zando-se mais freqüentemente de estratégias de aprendizagem e apresentando melhor rendimento acadêmico.

Um ponto recorrente destacado pelas pesquisas citadas é a necessidade de intensificar estudos que levem em conta os diferentes efeitos das metas de realização. No entanto ainda não existe um instrumento brasileiro capaz de discriminar as modalidades aproximação e evitação da meta performance. Diante disso, este trabalho foi proposto com os objetivos de investigar a estrutura fatorial da Escala de Sensibilidade às Diferentes Metas de Realização, sua precisão e eventuais correlações entre os fatores.

\section{Método}

\section{Participantes}

Participaram da coleta de dados 156 sujeitos, dos quais 93 (59,6\%) pertenciam ao curso de Psicologia, período diurno, de uma instituição de ensino superior (IES) da cidade de São Paulo, de nível socioeconômico mais alto, e 63 (40,4\%) pertenciam ao curso de Psicologia, período noturno, de uma IES de uma cidade do interior de São Paulo, de nível socioeconômico mais baixo. A amostra é predominantemente do sexo feminino (84,6\%). A idade dos sujeitos variou de 18 a 45 anos, com a média em 22,3 e desvio-padrão de 4,5.

\section{Instrumentos}

Foi utilizada a Escala de Sensibilidade às Diferentes Metas de Realização (Zenorini et al., 2003), composta por 17 itens que devem ser avaliados pelos sujeitos através da atribuição de pontos de 1 a 4, conforme concordem plenamente (4 pontos), concordem (3 pontos), discordem (2 pontos) ou discordem plenamente (1 ponto). A pontuação máxima possível é de 68 pontos e a mínima de 17 pontos.

\section{Procedimentos}

A aplicação dos instrumentos foi realizada pelos próprios pesquisadores, em situação de sala de aula,

82 após a informação sobre as características da pesquisa e a obtenção do consentimento livre e esclarecido dos sujeitos que concordaram em participar. Os dados foram armazenados em uma planilha eletrônica, e para atingir os objetivos propostos, foram realizadas análises de estatística descritiva, fatorial, correlacional e do coeficiente alfa de Cronbach. Foram considerados significativos os resultados que obtiveram $p<0,05$.

\section{Resultados}

A medida de adequação da amostra de Kaiser-Meyer-Olkin (KMO) foi de 0,81, que indicou uma boa adequação dos dados à análise fatorial. Ao lado disso, o teste de esfericidade de Bartlett foi significativo ao nível de $0,001 \quad\left(\chi^{2}=831,7 ; g l=136\right)$, mostrando que houve correlações suficientes entre as variáveis para o emprego da análise fatorial.

Os dados foram então submetidos a uma análise fatorial exploratória, com extração dos fatores por análise dos componentes principais e rotação varimax. Foram obtidos quatro fatores com eigenvalues acima de um, capazes de explicar 59,1\% da variância total. Na Tabela 1 são apresentadas as cargas fatoriais superiores a 0,30, os eigenvalues e os coeficientes alfa de Cronbach dos fatores.

Observou-se que o primeiro fator, com eigenvalue igual a 3,9, explicou 23,0\% da variância total por meio de nove itens, cujas cargas fatoriais foram de 0,427 a 0,827, e com uma consistência interna de 0,86. 0 segundo fator, com eigenvalue 3,0, explicou 17,6\% da variância total por meio de seis itens, com cargas fatoriais de 0,56 a 0,81, e com uma consistência interna de 0,78. 0 terceiro fator apresentou eigenvalue igual a 1,8, explicando 10,3\% da variância total, por meio de quatro itens, com cargas fatoriais que vão de -0,32 a 0,82, e consistência interna de 0,57. Finalmente, o quarto fator reuniu cinco itens, com cargas fatoriais que vão de -0,39 a 0,79 , resultando em um eigenvalue de 1,4, correspondente a $8,2 \%$ da variância total, e com uma consistência interna de 0,61.

As pontuações do fator 1, que teoricamente poderiam variar de 9 a 36, na realidade variaram de 9 a 34, abrangendo quase que toda a amplitude possível e com boa variabilidade $(4,6)$. A média desse fator $(15,4)$ 
Tabela 1. Cargas fatoriais, eigenvalues e coeficientes alfa de Cronbach.

\begin{tabular}{|c|c|c|c|c|}
\hline Itens & 1 & 2 & 3 & 4 \\
\hline 10. Sucesso na escola, para mim, é fazer as coisas melhor que a maioria da classe. & 0,827 & & & \\
\hline 12. É muito importante, para mim, fazer as tarefas melhor que os colegas. & 0,805 & & & \\
\hline 6. Na minha turma, eu quero me sair melhor que todos os demais. & 0,778 & & & \\
\hline 15. Gostaria de mostrar aos meus professores que eu sou mais inteligente do que os outros. & 0,757 & & & 0,437 \\
\hline $\begin{array}{l}\text { 11. A razão pela qual me dedico às tarefas escolares é para que os professores não fiquem pensando que } \\
\text { eu sei menos que os outros. }\end{array}$ & 0,630 & & & 0,336 \\
\hline 7. Uma razão importante pela qual eu estudo é que eu não quero passar vexame. & 0,581 & & & \\
\hline 3. Eu me sentiria bem se eu fosse o único que pudesse responder às perguntas do professor em classe. & 0,471 & & & \\
\hline 14. Uma razão pela qual eu faço minhas tarefas escolares é que eu gosto delas. & & 0,814 & & \\
\hline 13. Faço minhas tarefas escolares porque estou interessado nelas. & & 0,793 & & \\
\hline 8. Uma importante razão pela qual eu estudo pra valer é porque eu quero aumentar meus conhecimentos. & & 0,696 & & $-0,315$ \\
\hline $\begin{array}{l}\text { 4. Uma razão importante pela qual eu faço as tarefas escolares é porque eu gosto de aprender coisas } \\
\text { novas. }\end{array}$ & & 0,684 & $-0,316$ & \\
\hline $\begin{array}{l}\text { 2. Gosto dos trabalhos escolares com os quais eu aprendo algo, mesmo que cometa um bocado de } \\
\text { erros. }\end{array}$ & & 0,574 & & \\
\hline 5. Eu gosto mais das tarefas quando elas me fazem pensar. & & 0,556 & & $-0,394$ \\
\hline 1. É muito importante para mim que eu não apareça como burro na minha classe. & & & 0,821 & \\
\hline 16. Procuro sair-me bem nas tarefas escolares para que os outros não fiquem pensando que eu sou burro. & 0,427 & & 0,665 & \\
\hline 17. Um dos meus principais objetivos é nunca dar a impressão de que eu não consigo dar conta da tarefa. & 0,438 & & 0,553 & \\
\hline 9. Uma razão pela qual eu não participo da aula é evitar parecer ignorante. & & & & 0,790 \\
\hline Eigenvalue & 3,900 & 3,000 & 1,800 & 1,400 \\
\hline Coeficiente Alfa de Cronbach & 0,860 & 0,780 & 0,573 & 0,612 \\
\hline
\end{tabular}

ficou bem abaixo do ponto central da amplitude total (22,5). No fator 2, as pontuações podiam variar de 6 a 24, mas a amplitude obtida foi de 13 a 24, com média $(19,5)$ situada acima do ponto central da amplitude total (15), e baixa variabilidade $(2,7)$. No fator 3 , as pontuações podiam variar de 4 a 16, mas nesse grupo de sujeitos variaram de 4 a 14, com média (8,3) pouco abaixo do ponto central da amplitude total (10), e baixa variabilidade $(2,1)$. No fator 4 , as pontuações podiam variar de 5 a 20, mas variaram de 5 a 18, com média $(8,3)$ ficando bem abaixo do ponto central da amplitude total (12,5), e apresentando baixa variabilidade (2,1). A Tabela 2 apresenta os coeficientes de correlação de Pearson entre os quatro fatores obtidos.

Constatou-se que não houve correlação estatisticamente significativa entre os fatores 1 e 2. No entanto houve correlações significativas e positivas entre os fatores 1, 3 e 4, correlações significativas e negativas entre o fator 2 e o fator 3 , bem como entre o fator 2 e o fator 4.
Tabela 2. Coeficientes de correlação de Pearson entre os fatores.

\begin{tabular}{lccc}
\hline & \multicolumn{3}{c}{ Fator } \\
\cline { 2 - 4 } Fator & 1 & 2 & 3 \\
\hline 2 & $-0,140$ & \\
3 & $0,633^{*}$ & $-0,357^{*}$ & \\
4 & $0,598^{*}$ & $-0,551^{*}$ & $0,418^{*}$ \\
\hline
\end{tabular}

* Correlações significativas ao nível de 0,01.

\section{Discussão}

A análise fatorial revelou a possibilidade de agrupamento dos itens em quatro fatores, com a preservação de uma boa porcentagem da variância total (59,1\%). No entanto os dois primeiros fatores podem ser considerados como mais importantes, pois apresentaram eigenvalues mais significativos e foram responsáveis pela explicação de 41,6\% da variância total.

Os índices de fidedignidade dos dois primeiros fatores podem ser considerados razoáveis, enquanto os dois últimos são considerados insuficientes (Anastasi \& 
Urbina, 2000; Pasquali, 2003). Os dois primeiros fatores apresentaram os melhores resultados, indicando que possuem uma quantidade de variância verdadeira maior que a variância de erro. Nos fatores 3 e 4 isso não ocorreu, sugerindo que nesses fatores os itens ainda estejam avaliando outras coisas (desconhecidas) que não as desejáveis. A análise mais pormenorizada dos fatores pode ajudar a compreender a causa desse resultado.

O primeiro fator apresentou cinco itens com carga superior a 0,3 exclusivamente nesse fator, dois com carga também no quarto fator e dois com carga no terceiro fator. A análise de conteúdo dos itens exclusivos do fator 1 permite interpretá-lo como compatível à definição de meta performance aproximação (Elliot \& Harackiewicz, 1996; Skaalvik, 1997; Urdam, 1997), especialmente em relação ao desejo de se sair melhor do que os demais. A estrutura dessas frases envolve a descrição de uma ação (estudar, dedicar-se, mostrar aos outros) que tem como conseqüência um efeito positivo em termos de desempenho escolar (ser melhor do que os demais).

Seis itens apresentaram carga superior a 0,3 no segundo fator. Três deles apresentaram carga exclusivamente no segundo fator, um apresentou carga negativa e menor no terceiro fator e outros dois itens também apresentaram cargas negativas e menores no quarto fator. A análise do conteúdo desses itens sugere claramente a interpretação compatível com o conceito de meta aprender (Dweck \& Elliott, 1983; Dweck \& Leggett, 1988), com frases que descrevem uma ação (estudar, fazer tarefas escolares) realizada por algum tipo de envolvimento com a própria tarefa (gosto delas, quero aumentar meus conhecimentos, me fazem pensar, estou interessado nelas).

Os fatores 3 e 4 apresentaram algumas características semelhantes. Ambos se constituíram predominantemente por itens que também apresentaram cargas nos fatores 1 e 2, apresentaram apenas um item com carga elevada e exclusiva em cada um dos fatores, e a análise de conteúdo dos itens sugere a interpretação compatível com o conceito de meta performance evitação (Elliot \&Harackiewicz, 1996; Skaalvik, 1997; Urdam, 1997). Porém há uma distinção significativa a ser feita com relação às estruturas das frases. A que apresentou carga elevada e exclusivamente no fator 3 descreve uma situação em que o sujeito considera importante evitar 84 uma conseqüência negativa (parecer "burro" perante a classe), enquanto o item exclusivo do fator 4 descreve uma situação em que o sujeito evita uma ação (não participa da aula) para evitar uma conseqüência negativa (parecer ignorante).

A falta de um número maior de frases com estruturas semelhantes às exclusivas dos fatores 3 e 4 só nos permite levantar uma hipótese quanto ao acréscimo de um fator relacionado à meta performance evitação. Nesse caso, poderia haver um fator relacionado a considerar importante evitar conseqüência negativa ou agir para evitar conseqüência negativa (É muito importante para mim que eu não apareça como burro na minha classe), e outro relacionado à evitação da ação para evitar conseqüência negativa (Uma razão pela qual eu não participo da aula é evitar parecer ignorante). Outros estudos poderiam investigar essa questão específica, verificando o efeito que o acréscimo de itens com estruturas lingüísticas semelhantes a essas causaria tanto na estrutura fatorial quanto na fidedignidade desses fatores.

Outra observação interessante diz respeito aos itens que apresentaram cargas em mais de um fator. Quando isso acontece, o item, em geral, é considerado ruim por apresentar variância concomitantemente com dois fatores distintos. Portanto uma análise desses itens pode ser importante no sentido de mostrar o que deve ser evitado em uma futura revisão do instrumento.

Os itens que apresentaram carga no fator $1 \mathrm{e}$ também nos fatores 3 ou 4 possuem o mesmo tipo de estrutura lingüística do fator 3 , relacionada ao fato de que o estudante faz algo para evitar conseqüência negativa, ou considera importante evitar conseqüência negativa. Ou seja, esses itens parecem ter apresentado carga em ambos os tipos de meta performance porque uma parte da frase se refere à implementação de uma ação, enquanto outra parte se refere à evitação de uma conseqüência. Dito de outra forma, é possível que esses itens estejam interferindo negativamente na distinção entre os dois tipos de meta performance: aproximação e evitação. Ao lado disso, alguns itens que apresentaram carga mais elevada no fator 2 também apresentaram carga menor e negativa nos fatores 3 ou 4. Esse dado sugere a existência de correlação negativa entre meta aprender e meta performance-evitação.

De fato, as correlações entre os fatores mostram que não houve relação entre meta performance aproximação e meta aprender (F1 e F2), houve relação 
entre meta performance aproximação e meta performance evitação (F3 e F4), e também houve relação inversa entre meta aprender (F2) e os dois fatores relacionados à meta performance evitação (F3 e F4). Por este estudo não é possível saber qual tipo de meta ou qual associação entre as metas produz um bom desempenho escolar, já que esse não foi o objetivo deste trabalho, mas mostra que a meta aprender pode estabelecer uma relação mais estável e negativa com a performance evitação e não com a performance aproximação.

Esse achado teria uma implicação de natureza prática, já que alguns estudos prevêem que o melhor desempenho escolar seja fruto de uma associação entre a meta aprender e a meta performance aproximação (Bouffard et al., 1995; Harackiewicz et al., 1998), e no presente trabalho estaria mostrando que essa associação, embora desejável, não necessariamente ocorre de maneira natural. A forma como a Psicologia poderia ajudar os alunos a encontrar a medida adequada de associação entre essas duas metas é uma questão a ser respondida por meio da realização de outras pesquisas.

Com base nos índices de correlações observadas, os fatores 1, 3 e 4, todos relacionados à meta performance, estão altamente correlacionados entre si. Ao lado disso, o fator 2 (meta aprender) correlacionou-se negativamente com os fatores 3 e 4, relacionados à meta performance evitação. Ou seja, a meta performance evitação associa-se tanto com a meta performance aproximação (positivamente) quanto com a meta aprender (negativamente) mas as metas aprender e performance aproximação não se correlacionam entre si. Esse dado nos permite levantar a hipótese de que a meta performance evitação seja o elemento balizador entre as metas aprender e performance aproximação.

Nesse sentido, a associação da meta performance evitação com a meta performance aproximação poderia trazer resultados negativos, como sugere o estudo de Midgley et al. (2001), com a hipótese de que, diante do fracasso, o aluno orientado à meta performance aproximação poderia tender à meta performance evitação. Entretanto, a associação inversa (negativa) entre metaperformance evitação e meta aprender poderia estar associada ao prazer encontrado no estudo.

Uma vez que essas duas variáveis apareceram associadas negativamente, pode-se supor que o aumento da meta performance evitação, acompanhado pela diminuição da meta aprender, levaria à percepção do estudo como uma tarefa desprazível. Ao lado disso, a diminuição da meta performance evitação e o conseqüente aumento da meta aprender levariam à percepção do estudo como uma tarefa mais prazerosa. Essas questões também ficam como sugestões para investigação futura.

\section{Considerações Finais}

Considerando a relação entre as metas de realização e os fatores motivacionais que interferem no sucesso e no fracasso dos estudantes em sua vida escolar, e o impacto que a possibilidade de mensurar essas variáveis poderia ter no desenvolvimento de estratégias de intervenção psicoeducacional, a realização deste estudo foi proposta com os objetivos de: investigar a estrutura fatorial da Escala de Sensibilidade às Diferentes Metas de Realização, sua precisão e as correlações entre os fatores.

Os resultados encontrados confirmam a estruturação das metas de realização em meta aprender, meta performance aproximação e meta performance evitação, e acrescentam a sugestão de que a meta performance evitação possa ser subdivida em um fator relacionado a "considerar importante a evitação de conseqüência negativa" (fator 3) e outro relacionado a"evitar uma ação para evitar sua conseqüência negativa" (fator 4). Diante disso, sugere-se a realização de outro estudo para a inclusão de itens com essas estruturas e a eliminação de itens com a estrutura "fazer algo para evitar conseqüência negativa", já que esse tipo de estrutura lingüística parece interferir negativamente na distinção entre os aspectos aproximação e evitação da meta performance.

Dessa forma, este trabalho aponta caminhos possíveis para a continuidade do desenvolvimento de um instrumento para avaliação das metas de realização. Deve ser compreendido como uma etapa do processo e não como seu resultado final. Mostra como o trabalho de construção de um instrumento de avaliação psicológica é árduo e complexo, e, muitas vezes, deve ser realizado em etapas para que os problemas possam ser superados. 


\section{Referências}

Ames C., \& Archer, J. (1988). Achievement goals in the classroom: student's learning strategies and motivational processes. Journal of Educational Psychology, 80 (3), 260-267.

Ames, C. (1992). Classrooms: goals, structures, and student motivation. Journal of Education Psychology, 84 (3), 261-271.

Anastasi, A., \& Urbina, S. (2000). Testagem psicológica. Porto Alegre: Artes Médicas.

Anderman, E. M., \& Maehr, M. L. (1994). Motivation and schooling in the middle grades. Review of Education Research, 64 (2), 287-309.

Barron, K. E., \& Haracckiewicz, J. M. (2000). Achievement goals and optimal motivation: a multiple goals approach. In I. C. Sansone \& J. M. Harackiewicz (Orgs.), Intrinsic and extrinsic motivation: the search for optimal motivation and performance (pp.229-254). New York: Academic Press.

Barron, K. E., \& Haracckiewicz, J. M. (2001). Achievement goals and optimal motivation: testing multiple goal models. Journal of Personality and Social Psychology, 80 (5), 706-722.

Boruchovitch, E., \& Costa, E. R. (2001). O impacto da ansiedade no rendimento escolar e na motivação de alunos. In E. Boruchovitch \& J. A. A. Bzuneck (Orgs.), A motivação do aluno. Contribuições dapsicologia contemporânea (pp.134147). Petrópolis: Vozes.

Boruchovitch, E., \& Martini, M. L. (1997). As atribuições de causalidade para o sucesso e o fracasso escolar e a motivação para a aprendizagem de crianças brasileiras. Arquivos Brasileiros de Psicologia, 49 (3), 59-71.

Bouffard, T., Boisvert, J., Vezeau, C., \& Larouche, C. (1995). The impact of goal orientation on self-regulation and performance among college students. British Journal of Educational Psychology, 65, 317-29.

Bzuneck, A. (1999). Uma abordagem sócio-cognitivista à motivação do aluno: a teoria de metas de realização. Psico-USF, 4 (2), 51-66.

Bzuneck, J. A. (2001). A motivação do aluno: aspectos introdutórios. In E. Boruchovitch \& J. A. A Bzuneck (Orgs.), A motivação do aluno. Contribuições da psicologia contemporânea (pp.9-36). Petrópolis: Vozes.

Cardoso, L. R. (2002). Uso de estratégias de aprendizagem e suas relações com metas de realização: um estudo no ensino superior. Dissertação de mestrado em Educação nãopublicada, Universidade Estadual de Londrina.

Duda, J. L., \& Nicholls, J. G. (1992). Dimensions of achievement motivation in school work and sport. Journal of Education Psychology, 84 (3), 290-299.

Dweck, C. S., \& Elliott, E. S. (1983). Achievement Motivation. In E. H. Hetheringtton \& P. H. Mussen. Handbook of child Psychology: socialization, personality and social development (pp. 643-692). New York: Wiley.

Dweck, C. D., \& Leggett, E. L. (1988). A social-cognitive approach to motivation and personality. Psychological Review, 95 (2), 256-273.
Elliot, E. S., \& Dweck, C. S. (1988). Goals: an approach to motivation and achievement. Journal of Personality and Social Psychology, 54 (1), 5-12.

Elliot, A. J., \& Harackiewicz, J. M. (1996). Approach and avoidance achievement goals and intrinsic motivation: a mediational analysis. Journal of Personality and Social Psychology, 70 (3), 461-475.

Elliot, A. J. (1999). Approach and avoidance motivation and achievement goals. Educational Psychologist, 34, 169-189.

Gombi, R. B. O. (1999). Orientações motivacionais e emprego de estratégias de aprendizagem no estuda da disciplina de Sociologia em cursos superiores. Dissertação de mestrado em Educação não-publicada, Universidade Estadual de Londrina.

Grant, H., \& Dweck, C. S. (2003). Clarifying achievement goals and their impact. Journal of Personality and Social Psychology, 85 (3), 541-553.

Guimarães, S. E. R. (2001). Motivação intrínseca, extrínseca e o uso de recompensas. In E. Boruchovich \& J. A. A. Bzuneck (Orgs.), A motivação do aluno: contribuições da Psicologia contemporânea (pp. 37-57). Petrópolis: Vozes.

Guimarães, S. E. R. (2003). Avaliação do estilo motivacional do professor: adaptação e validação de um instrumento. Tese de doutorado não-publicada, Universidade Estadual de Campinas.

Guimarães, S. E. R., Bzuneck, J. A. A., \& Sanches, S. F. (2002). Psicologia educacional nos cursos de licenciatura: a motivação dos estudantes. Psicologia Escolare Educacional, $6(1), 11-19$.

Harackiewicz, J. M., Barron, K. E., \& Elliot, A. J. (1998). Rethinking achievement goals: when are they adaptive for college students and why? Educational Psychologist, $33,1-21$.

Harackiewicz, J. M., Barron, K. E., Pintrich, P. R, Elliot, A., \& Thash, T. M. (2002). Revision of achievement goal theory: necessary and illuminating. Journal of Educational Psychology, 94 (3), 638-645.

Maehr, M. L., \& Midgley. C. (1991). Enhancing student motivation: a school wide approach. Educational Psychologist, 26 (3/4), 399-427.

McGregor, H. A., \& Elliot, A. J. (2002). Achievement goals as predictors of achievement-relevant processes prior to task engagement. Journal of Educational Psychology, 94 (2), 381-395.

Meece, J. L., Blumenfeld, P. C., \& Hoyle, R. H. (1988). Student's goal orientation and cognitive engagement in classroom activities. Journal of Educational Psychology, 80 (4), 514-523.

Midgley, C., Kaplan, A., \& Middleton, M. (2001). Good for what, for whom, under what circumstances, and at what cost? Journal of Educational Psychology, 93 (1), 77-86.

Midgley, C., Kaplan, A., Middleton, M., Maehr, M. L., Urdan, T., Anderman, L. H., Anderman, E., \& Roeser, R. (1998). The development and validation of scales assessing students' achievement goals orientations. Contemporary Educational Psychology, 23, 113-131. 
Nicholls, J. G. (1984). Conceptions of ability and achievement motivation. In R. Ames \& C. Ames (Orgs.), Research on motivation in education: student motivation (pp.39-73). New York: Academic Press.

Pasquali, L. (2003). Psicometria: teoria dos testes na Psicologia e na Educação. Petrópolis: Vozes.

Pintrich, P. R., \& Schunk, D. H. (2002). Motivation in education: theory research and applications. New Jersey: Pearson Education Inc.

Pintrich, P. R. (2000). Multiple goals, multiple pathways: the role of goal orientation in learning and achievement. Journal of Educational Psychology, 92 (3), 544-555.

Ruiz, V. M. (2005). Aprendizagem em universitários: variáveis motivacionais. Tese de doutorado não-publicada, Pontifícia Universidade Católica de Campinas.

Skaalvick, E. M. (1997). Self-enhancing and self-defeating ego orientation: relations with task and avoidance orientation, achievement, self-perceptions, and anxiety. Journal of Educational Psychology, 89 (1), 71-81.

Urdan, T. C. (1997) Achievement goal theory: Past results, future diretions. In M. L. Maehr \& P. R. Pintrich (Orgs.), Advances in motivation and achievement (pp.99-141). Greenwich, CT.: JAl Press.

Zenorini, R. P. C., \& Santos, A. A. A. (2003). A motivação e a utilização de estratégias de aprendizagem em universitários. In E. Mercuri \& S. A. J. Polydoro. Estudante universitário: características e experiências de formação (pp. 67-86). Taubaté: Cabral Editora.

Zenorini, R. P. C., Santos, A. A. A., \& Bueno, J. M. H. (2003). Escala de avaliação das metas de realização: estudo preliminar de validação. Avaliação Psicológica, 2 (2), 165-173.

Recebido em: 25/5/2006

Aprovado em: 16/8/2006 
